Pacific

Journal of

Mathematics

\title{
DIRICHLET FORMS AS BANACH ALGEBRAS AND
} APPLICATIONS

\section{FABIO CIPRIANI}




\title{
DIRICHLET FORMS AS BANACH ALGEBRAS AND APPLICATIONS
}

\author{
FABIO CIPRIANI
}

Dedicated to Gianfausto Dell'Antonio on his seventieth birthday.

\begin{abstract}
We study regular Dirichlet forms on locally compact Hausdorff spaces $X$ in the framework of the theory of commutative Banach algebras. We prove that, suitably normed, the Dirichlet algebra $\mathscr{B}_{e}=C_{0}(X) \cap \mathscr{F}_{e}$ of continuous functions vanishing at infinity in the extended domain $\mathscr{F}_{e}$ of a Dirichlet form $(\mathscr{E}, \mathscr{F})$ is a semisimple Banach algebra. This implies that two strongly local Dirichlet forms $\left(\mathscr{E}_{1}, \mathscr{F}_{1}\right),\left(\mathscr{E}_{2}, \mathscr{F}_{2}\right)$ are quasi-equivalent (that is, $c^{-1} \mathscr{E}_{1} \leq \mathscr{E}_{2} \leq$ $c \mathscr{E}_{1}$ for some $c>0$ ) if and only if they have the same domain.

We describe the ideal structure of $\mathscr{B}_{e}$, showing that the algebraic $K$ theory $K_{*}\left(\mathscr{B}_{e}\right)$ of the Dirichlet algebra $\mathscr{B}_{e}$ is isomorphic to the topological $K$-theory $K^{*}(X)$. This allows the construction of Dirichlet structures on (sections of) finite-dimensional, locally trivial vector bundles over $X$.
\end{abstract}

\section{Introduction}

We study Dirichlet forms from the viewpoint of the theory of commutative Banach algebras; this approach can be compared to the one illustrated in [Cipriani and Sauvageot 2003], where it was shown how the study of regular Dirichlet forms can be regarded as the study of closed derivations on algebras of continuous functions, taking values in Hilbert modules.

In this section we consider some motivating questions concerning quadratic forms, which will be answered using algebraic tools. A summary of the rest of the paper can be found at the end of Section 2.

Question 1. To what extent does the Dirichlet integral

$$
\mathscr{E}[a]=\int_{\mathbb{R}^{n}}|\nabla a|^{2} d m
$$

on $L^{2}\left(\mathbb{R}^{n}, m\right)$ depend on its domain, that is, on the Sobolev space $H^{1,2}\left(\mathbb{R}^{n}\right)$ where $\mathscr{E}$ is finite?

MSC2000: primary 31C25; secondary 46J10, 46J20, 60J45.

Keywords: Dirichlet form, Banach algebra, $K$-theory. 
The energy integral (1-1) can be considered the prototype of a (regular, strongly local) Dirichlet form; in addition to its lower semicontinuity, it has the contraction property

$$
\mathscr{E}[a \wedge 1] \leq \mathscr{E}[a]
$$

which characterizes, by definition, Dirichlet forms among the lower semicontinuous ones.

Question 2. Can we describe all (regular, strongly local) Dirichlet forms on $L^{2}\left(\mathbb{R}^{n}, m\right)$ that are finite exactly on the Sobolev space $H^{1,2}\left(\mathbb{R}^{n}\right)$ ?

For example, let us consider the (uniformly quasiconformal) perturbation of the Dirichlet integral given by quadratic forms of the type

$$
\mathscr{E}_{g}[a]=\sum_{i, j=1}^{n} \int_{\mathbb{R}^{n}} g^{i, j}(x) \partial_{i} a(x) \partial_{j} a(x) m(d x),
$$

associated to symmetric matrices of measurable function, subject to a uniform ellipticity condition $0<\lambda \leq\left[g^{i, j}\right]_{i, j=1}^{n} \leq \Lambda<+\infty$; since all the forms of type (1-2) are Dirichlet forms, finite exactly on the same space, we may ask:

Question 3. Do the forms of the type above exhaust the class of regular, strongly local Dirichlet forms that are finite precisely on the Sobolev space $H^{1,2}\left(\mathbb{R}^{n}\right)$ ?

The answer, in the affirmative, relies, among other things, on the fact that the space $C_{0}\left(\mathbb{R}^{n}\right) \cap H^{1,2}\left(\mathbb{R}^{n}\right)$ of continuous functions vanishing at infinity and having finite Dirichlet integral is a uniformly dense subalgebra of $C_{0}\left(\mathbb{R}^{n}\right)$, which is also a semisimple algebra with respect to a suitable Banach norm, stronger than the uniform one. This and other algebras with similar properties on locally compact Hausdorff spaces, called in this work Dirichlet algebras (not to be confused with those considered in [Wermer 1960]), represent the objects of our present investigation. The special case of the classical Dirichlet form on the unit interval $[0,1] \subset \mathbb{R}$ was investigated by G. Shilov [1947] (see [Rickart 1960, Appendix §2]).

We conclude this introduction noticing that the algebraic point of view dates back to the seminal work on the subject by A. Beurling and J. Deny [1959], where they indicated, although without proof and in the very special case of the Dirichlet integral on a line segment, that bounded continuous functions of finite energy form a Banach algebra and that all of its prime ideals are primitive. In addition one can detect the algebraic flavor at several stages along the development of the theory: we just mention, in Silverstein's boundary theory [1976] of Dirichlet forms, the definition of extension of a Dirichlet form and its similarity with the algebraic approach of Gelfand, Raikov and Shilov [Gelfand et al. 1960] to the compactification of completely regular spaces, and the use of the Gelfand spectrum of various algebras in M. Fukushima's regularization technique [Fukushima et al. 1994]. 


\section{Dirichlet forms and Dirichlet algebras}

Unless explicitly stated, the symbol $(X, m)$ will denote a locally compact, metrizable space with countable base, endowed with a positive Radon measure $m$ with full topological support (that is, strictly positive on open sets).

As is customary, $C_{0}(X)$ will denote the algebra of continuous functions vanishing at infinity on $X, L^{\infty}(X, m)$ the algebra of (classes of) $m$-essentially bounded functions on $X$, and $L^{2}(X, m)$ the Hilbert space of (classes of) square-integrable functions on $X$.

The standing assumptions on $(X, m)$ imply that $C_{0}(X) \cap L^{2}(X, m)$ can be viewed both as a subspace of $C_{0}(X)$ as well as of $L^{2}(X, m)$, that these embeddings are dense both in the uniform norm as well as in the $L^{2}$-topologies, and that $C_{0}(X)$ is weak*-dense in $L^{\infty}(X, m)$.

Our aim is to analyze from an algebraic point of view the following class of functionals (see [Albeverio 2003; Beurling and Deny 1959; Fukushima et al. 1994; Ma and Röckner 1992]).

Definition 2.1. A Dirichlet form on $(X, m)$ is a quadratic, lower semicontinuous functional

$$
\mathscr{E}: L^{2}(X, m) \rightarrow[0,+\infty]
$$

which is finite on a dense set and satisfies

$$
\mathscr{E}[a \wedge 1] \leq \mathscr{E}[a] \quad a \in L^{2}(X, m) .
$$

The domain $\mathscr{F}$, called the Dirichlet space, is, by definition, the subspace where $\mathscr{E}$ is finite

$$
\mathscr{F}:=\left\{a \in L^{2}(X, m): \mathscr{E}[a]<+\infty\right\} .
$$

In the following will have occasion to denote by $\mathscr{E}(\cdot, \cdot)$ the symmetric sesquilinear form on $\mathscr{F}$ associated by polarization to $\mathscr{E}[\cdot]$.

Remark 2.2. It was proved in [Cipriani 1997, Proposition 4.10(i)] that this definition of a Dirichlet form is equivalent to the usual one [Fukushima et al. 1994], where $(2-1)$ is replaced by $\mathscr{E}[0 \vee a \wedge 1] \leq \mathscr{E}[a]$, thanks to the lower semicontinuity of $\mathscr{E}$.

Remark 2.3. All the linear spaces of functions considered in this paper are on the field of complex numbers. Correspondingly, we tacitly assume that the Dirichlet forms considered are real in the sense that $\mathscr{E}[\bar{a}]=\mathscr{E}[a]$ for all $a$ in the Dirichlet space $\mathscr{F}$. This is equivalent to assuming that if $a$ is in $\mathscr{F}$ then its real and imaginary parts, $\operatorname{Re} a$ and $\operatorname{Im} a$, also belongs to $\mathscr{F}$ and $\mathscr{E}[a]=\mathscr{E}[\operatorname{Re} a]+\mathscr{E}[\operatorname{Im} a]$. This may explains why in Definition 2.1 only real functions are involved.

Recall that Dirichlet forms on $L^{2}(X, m)$ are in one-to-one correspondence with the class of symmetric Markov semigroups on $L^{2}(X, m)$, i.e., those symmetric, 
strongly continuous, positivity-preserving contraction semigroups on $L^{2}(X, m)$ that extend to strongly continuous, positivity-preserving contraction semigroups on $L^{p}(X, m)$ for all $p \in[0,+\infty)$ and to a weakly continuous, positivity-preserving contraction semigroup on $L^{\infty}(X, m)$. See [Albeverio 2003; Fukushima et al. 1994; Ma and Röckner 1992].

Besides $(\mathscr{E}, \mathscr{F})$ it is important to consider the extended Dirichlet space $\left(\mathscr{E}, \mathscr{F}_{e}\right)$, in view of its stronger stability. Here $\mathscr{F}_{e}$ is the space of those $m$-a.e. finite Borel functions $a$ on $X$ for which there exists a sequence $\left\{a_{n} \in \mathscr{F}: n \in \mathbb{N}\right\}$ such that $a=\lim _{n \rightarrow \infty} a_{n} m$-a.e., $\mathscr{E}\left[a_{n}-a_{m}\right] \rightarrow 0$ as $n, m \rightarrow \infty$, and then $\mathscr{E}[a]$ is defined as the limit $\mathscr{E}[a]=\lim _{n \rightarrow \infty} \mathscr{E}\left[a_{n}\right]$.

Notice that, in general, one has $\mathscr{F}_{F}=\mathscr{F}_{e} \cap L^{2}(X, m)$, so that $\mathscr{F}_{F} \mathscr{F}_{e}$ if the measure is finite. Moreover, as a consequence of the Potential Theory of Dirichlet spaces, one can prove that even if, in general, $\mathscr{F}_{e}$ cannot be embedded in $L^{2}(X, m)$, it can be always embedded in $L^{1}(X, \mu)$ for a suitable measure $\mu$. A Dirichlet form is said to be recurrent if $1 \in \mathscr{F}_{e}$ and $\mathscr{E}[1]=0$. It is said to be transient if $a \in \mathscr{F}_{e}$ and $\mathscr{E}[a]=0$ imply $a=0$.

For example, the Dirichlet integral (1-1) is recurrent if and only if the dimension of the Euclidean space is 1 or 2, and it is transient if and only if the dimension is at least 3.

We now introduce the main objects of our investigation: these will be natural function algebras associated to Dirichlet spaces.

Definition 2.4. Let $(\mathscr{E}, \mathscr{F})$ be a Dirichlet form on $L^{2}(X, m)$ and consider the following spaces:

(i) the Dirichlet algebra

$$
\mathscr{B}:=C_{0}(X) \cap \mathscr{F} ;
$$

(ii) the extended Dirichlet algebra

$$
\mathscr{B}_{e}:=C_{0}(X) \cap \mathscr{F}_{e} ;
$$

(iii) the weak Dirichlet algebra

$$
\mathscr{A}:=L^{\infty}(X, m) \cap \mathscr{F}
$$

(iv) the extended weak Dirichlet algebra

$$
\mathscr{A}_{e}:=L^{\infty}(X, m) \cap \mathscr{F}_{e} .
$$

These are vector spaces that satisfy the obvious inclusion relations:

$$
\mathscr{A} \subseteq \mathscr{A}_{e}, \quad \mathscr{B} \subseteq \mathscr{B}_{e}, \quad \mathscr{B} \subseteq \mathscr{A}, \quad \mathscr{B}_{e} \subseteq \mathscr{A}_{e} .
$$


The first relevant aspect concerning the structure of Dirichlet spaces was discovered by Beurling and Deny in their first paper on the subject [1959]: as the chosen names suggest, these spaces are function algebras.

Proposition 2.5. Let $(\mathscr{E}, \mathscr{F})$ be a Dirichlet form on $L^{2}(X, m)$. Then $\mathscr{A}, \mathscr{A}_{e}, \mathscr{B}$ and $\mathscr{B}_{e}$ are function algebras and

$$
\sqrt{\mathscr{E}[a b]} \leq \sqrt{\mathscr{E}[a]}\|b\|_{\infty}+\|a\|_{\infty} \sqrt{\mathscr{E}[b]} \text { for } a, b \in \mathscr{A}_{e} .
$$

Even if the theory of Dirichlet forms can be developed in fairly general measured spaces $(X, m)$, a more satisfactory and rich theory take places when $(X, m)$ are topological data. In this setting the key property on which this "topological" theory relies is the following one (see [Fukushima et al. 1994]).

Definition 2.6. A Dirichlet form $\mathscr{E}$ is said to be regular if its Dirichlet algebra $\mathscr{B}:=C_{0}(X) \cap \mathscr{F}$ is a core for $\mathscr{E}$ which is dense in $C_{0}(X)$, with respect to the uniform topology (notice also that alternative definitions are also considered in literature).

The core property can be expressed by saying that the closed quadratic form $(\mathscr{E}, \mathscr{F})$ is the closure of its restriction $(\mathscr{E}, \mathscr{B})$ or by saying that $\mathscr{E}$ is the relaxation (in the sense of the $\Gamma$-convergence of functionals) of the functional $\mathscr{E}^{0}: L^{2}(X, m) \rightarrow$ $[0,+\infty]$ which coincides with $\mathscr{E}$ in $\mathscr{B}$ but takes value $+\infty$ in the complement of $\mathscr{B}$ in $L^{2}(X, m)$.

Remark 2.7. The definition above is suitable in studying Dirichlet forms on locally compact Hausdorff spaces; otherwise, the space $C_{0}(X)$ can be very poor in topological information and may even vanish, as in the case of infinite-dimensional topological vector spaces (by Tychonoff's theorem). However, thanks to regularization procedures (see [Albeverio 2003; Fukushima et al. 1994; Ma and Röckner 1992]), one can apply the techniques we are going to develop to Dirichlet forms on much more general Hausdorff topological spaces.

Among the striking consequences of the above regularity hypothesis, we recall the following ones:

- $\mathscr{E}$ gives rise to a strongly subadditive Choquet capacity and a potential theory on $X$ which are the main tools to construct a canonical class of $m$-symmetric Hunt processes $\mathbf{M}=\left(\Omega, \mathcal{M}, X_{t}, P_{x}\right)$ on $X$ whose energy forms coincide with $\mathscr{E}$ (see [Fukushima et al. 1994]). These processes coincide outside a set of zero capacity. If $\Delta$ denotes the nonnegative, self-adjoint operator whose quadratic form is $\mathscr{E}$ and $\left\{e^{-t \Delta}: t \geq 0\right\}$ its associated Markov semigroup on $L^{2}(X, m)$, then the connection with the Hunt process can be expressed through the formula

$$
\left(T_{t} a\right)(x)=\mathbb{E}_{m}\left(a\left(X_{t}\right)\right),
$$


valid for all $t \geq 0$ and for quasi-every $x \in X$, with respect to the appropriate capacity. On the other hand, $m$-symmetric Hunt processes give rise to Dirichlet forms.

- $\mathscr{E}$ gives rise to a canonical differential calculus on $X$ (see [Cipriani and Sauvageot 2003]): there exists a canonical $L^{2}$-closable derivation $\partial: \mathscr{B} \rightarrow \mathscr{H}$ into a Hilbert space $\mathscr{H}$ endowed with a structure of symmetric $C_{0}(X)$-bimodule, such that

$$
\mathscr{E}[a]=\|\partial a\|_{\mathscr{H}}^{2} \quad \text { for } a \in \mathscr{B}
$$

in other words, the nonnegative, self-adjoint operator $\Delta$ on $L^{2}(X, m)$ associated to $\mathscr{E}$ can always be represented as the divergence of a derivation

$$
\Delta=\partial^{*} \circ \partial
$$

the Hilbert module $\mathscr{H}$ and the derivation $\partial$ are generalizations of the space of the square-integrable vector fields on a Riemannian manifold $X$ and the associated gradient respectively.

- The Dirichlet algebra $\mathscr{B}=C_{0}(X) \cap \mathscr{F}$ retains all the topological information of $X$ (see [Cipriani and Sauvageot 2003, Remark 7.6]): for example, the derivation $\partial$ is closed with respect to the uniform topology so that the algebraic $K$-theory of $\mathscr{B}$ is isomorphic to the topological $K$-theory of $X$ :

$$
K_{*}(\mathscr{B}) \cong K^{*}(X)
$$

by the methods developed in this paper, we give, in Section 4, an alternative proof of the corresponding result for the extended Dirichlet space $\mathscr{B}_{e}$.

- $\mathscr{E}$ can be canonically represented by a family $\left\{\mu_{\langle a\rangle}: a \in \mathscr{F}\right\}$ of energy measures (see [Cipriani and Sauvageot 2003; Lejan 1978; Fukushima et al. 1994]): these are positive Radon measures on $X$ which represent the Dirichlet form as $\mathscr{E}[a]=$ $\mu_{\langle a\rangle}(X)$ for $a \in \mathscr{F}$. They are characterized by the identities

$$
\begin{array}{ll}
\int_{X} b d \mu_{\langle a\rangle}=2 \mathscr{E}(a b, a)-\mathscr{E}\left(a^{2}, b\right) & \text { for } a, b \in \mathscr{B}, \\
\int_{X} b d \mu_{\langle a\rangle}=(b \partial a, \partial a) \mathscr{H} & \text { for } a, b \in \mathscr{B} .
\end{array}
$$

- In case $(\mathscr{E}, \mathscr{F})$ is strongly local in the sense that

$$
\mathscr{E}[a+b]=\mathscr{E}[a]+\mathscr{E}[b]
$$

whenever $a, b \in \mathscr{B}$ and $a$ is constant in a neighborhood of the support of $b$, the canonical differential calculus $(\partial B, \mathscr{B}, \mathscr{H})$ is local (see [Cipriani and Sauvageot 2003]) in the sense that $\mathscr{H}$ is a direct integral $\int_{X}^{\oplus} \mathscr{H}_{x}$ of $C_{0}(X)$-monomodules and the derivation $\partial$ can be expressed as a direct integral $\int_{X}^{\oplus} \partial_{x}$ of derivations which 
satisfy the Leibniz property

$$
\partial_{x}(a b)=\left(\partial_{x} a\right) b(x)+a(x)\left(\partial_{x} b\right) \quad \text { for } a, b \in \mathscr{B} \text { and a.e. } x \in X .
$$

We now summarize the paper. The main result of Section 3 is that the extended Dirichlet algebras $\mathscr{A}_{e}, \mathscr{B}_{e}$ are semisimple Banach algebras with respect to the sum of the uniform and energy norms so that they have a unique Banach algebra topology. In Section 4 we prove that the spectrum of the Dirichlet algebra $\mathscr{B}_{e}$ is homeomorphic with $X$, that the Dirichlet algebra $\mathscr{B}_{e}$ is stable under holomorphic functional calculus and that its algebraic $K$-theory $K_{*}\left(\mathscr{B}_{e}\right)$ is isomorphic to the topological $K$-theory $K^{*}(X)$ of $X$. This last result is then applied to show how a Dirichlet form on locally compact Hausdorff space $X$ gives rise, in a natural way, to modules of Dirichlet sections of finite-dimensional, locally trivial vector bundles over $X$. In Section 5 we prove that the Dirichlet algebra $\mathscr{B}_{e}$ is a completely regular semisimple Banach algebra; this implies the existence of partitions of the identity and the local (reconstruction) theorem. In Section 6 we complete the study of the structure of the ideals of the Dirichlet algebra $\mathscr{B}_{e}$ showing that every closed ideal is the kernel of its support and proving that $\mathscr{S}_{e}$ is an $N$-algebra and an $N^{*}$-algebra too. In Section 7 we use these results to answer the questions posed in the introduction and their generalizations for arbitrary strongly local, regular Dirichlet forms. In Section 8 we make some final considerations on the approach to Dirichlet forms developed here and formulate natural problems suggested by the algebraic point of view.

\section{Dirichlet spaces as Banach algebras}

The aim of this section is to introduce, for a fixed regular Dirichlet form $(\mathscr{E}, \mathscr{F})$, a natural norm on the Dirichlet algebras $\mathscr{A}_{e}, \mathscr{B}_{e}$ making them Banach algebras; we then prove that under this norm these are semisimple Banach algebras, so that all other possible Banach algebra structures on them are actually equivalent to the natural one. We will denote by $\|\cdot\|_{\infty}$ the uniform norm in $L^{\infty}(X, m)$.

Proposition 3.1. The function spaces $\mathscr{A}_{e}$ and $\mathscr{B}_{e}$, endowed with the norm

$$
\|a\|:=\|a\|_{\infty}+\sqrt{\mathscr{E}[a]} \text { for } a \in \mathscr{A}_{e},
$$

are complex Banach algebras.

Proof. Let $\left\{a_{n} \in \mathscr{A}_{e}: n \in \mathbb{N}\right\}$ be a Cauchy sequence in $\mathscr{A}_{e}$. Then it converges uniformly to an element $a \in L^{\infty}(X, m)$. By taking a subsequence, we can assume that it converges pointwise $m$-a.e. on $X$. Since the sequence is also $\mathscr{E}$-Cauchy, this implies that $a \in F_{e}$, so $a \in \mathscr{A}_{e}$. Since $C_{0}(X)$ is a norm closed subspace in $L^{\infty}(X, m)$, the same argument implies that $\mathscr{B}_{e}$ is norm closed in $\mathscr{A}_{e}$. 
Let us consider now the pointwise defined product $(a, b) \mapsto a b$ and the involution given by the pointwise conjugation $a \mapsto \bar{a}$ on the Banach algebras $C_{0}(X)$ and $L^{\infty}(X, m)$.

Proposition 3.2. The Dirichlet spaces $\mathscr{A}_{e}$ and $\mathscr{P}_{e}$, endowed with the algebraic structures above, are involutive complex Banach algebras. $\mathscr{A}_{e}$ is unital (i.e., $1 \in \mathscr{A}_{e}$ and $\|1\|=1)$ if and only if $(\mathscr{E}, \mathscr{F})$ is recurrent, and $\mathscr{B}_{e}$ is unital if and only if $X$ is compact and $(\mathscr{E}, \mathscr{F})$ is recurrent. The Dirichlet algebras $\mathscr{A}_{e}$ and $\mathscr{B}_{e}$ are never $C^{*}$-algebras unless $\mathscr{E}$ vanishes identically.

Proof. Since, by assumption, the Dirichlet form is real, the conjugation is a well defined operation on $\mathscr{A}_{e}, \mathscr{B}_{e}$ and $\|\bar{a}\|=\|a\|$ for all $a$ belonging to $\mathscr{A}_{e}$ or $\mathscr{P}_{e}$.

It is well known that $\mathscr{A}_{e}$ is closed under this product and that

$$
\sqrt{\mathscr{E}[a b]} \leq \sqrt{\mathscr{E}[a]}\|b\|_{\infty}+\|a\|_{\infty} \sqrt{\mathscr{E}[b]} \text { for } a, b \in \mathscr{A}_{e}
$$

(see [Fukushima et al. 1994]). This implies that for all $a, b \in \mathscr{A}_{e}$, we have

$$
\begin{aligned}
\|a b\|=\|a b\|_{\infty}+\sqrt{\mathscr{E}[a b]} & \leq\|a\|_{\infty}\|b\|_{\infty}+\sqrt{\mathscr{E}[a]}\|b\|_{\infty}+\|a\|_{\infty} \sqrt{\mathscr{E}[b]} \\
& \leq\left(\|a\|_{\infty}+\mathscr{E}[a]\right)\left(\|b\|_{\infty}+\mathscr{E}[b]\right) \\
& =\|a\|\|b\|,
\end{aligned}
$$

which proves that $\mathscr{A}_{e}$ and $\mathscr{B}_{e}$ are complex Banach algebras with involutions.

Since the constant function 1 always belongs to $L^{\infty}(X, m)$, we see that $\mathscr{A}_{e}$ is an involutive Banach algebra with unit if and only if $1 \in \mathscr{F}_{e}$ and $\mathscr{E}[1]=0$. Likewise, since $1 \in C_{0}(X)$ if and only if $X$ is compact, $\mathscr{B}_{e}$ is an involutive unital Banach algebra if and only if $X$ is compact, $1 \in \mathscr{F}_{e}$ and $\mathscr{E}[1]=0$.

Assume that $\mathscr{A}_{e}$ and $\mathscr{B}_{e}$ are a $C^{*}$-algebra: this means that $\|\bar{a} a\|=\|a\|^{2}$ for all $a$ in $\mathscr{A}_{e}$ and all $a$ in $\mathscr{B}_{e}$. Using (3-2) and the fact that $\mathscr{E}$ is real, we have

$$
\begin{aligned}
\|a\|_{\infty}^{2}+2\|a\|_{\infty} \sqrt{\mathscr{E}[a]}+\mathscr{E}[a] & =\|a\|^{2}=\|\bar{a} a\|=\|\bar{a} a\|_{\infty}+\sqrt{\mathscr{E}[\bar{a} a]} \\
& \leq\|a\|_{\infty}^{2}+2\|a\|_{\infty}^{2} \sqrt{\mathscr{E}[a]},
\end{aligned}
$$

which implies that $\mathscr{E}[a] \leq 0$ for all $a$ in $\mathscr{A}_{e}$ and all $a$ in $\mathscr{B}_{e}$. Hence, if $\mathscr{A}_{e}\left(\right.$ resp. $\left.\mathscr{B}_{e}\right)$ is a $C^{*}$-algebra, $\mathscr{E}$ vanishes identically on $\mathscr{A}$ (resp. $\left.\mathscr{B}\right)$. Since $\mathscr{A}$ is always a form core for $(\mathscr{E}, \mathscr{F})$, we have that the Dirichlet form is identically zero. The same implication holds true for $\mathscr{B}_{e}$ since the Dirichlet form is regular.

Theorem 3.3. The spectral radius $\rho(a)$ of an element a of the involutive complex Banach algebra $\mathscr{A}_{e}$ (resp. $\mathscr{B}_{e}$ ) coincides with its spectral radius $\|a\|_{\infty}$ as an element of the involutive complex Banach algebra $L^{\infty}(X, m)\left(\right.$ resp. $\left.C_{0}(X)\right)$.

In particular, the Dirichlet algebras $\mathscr{A}_{e}$ and $\mathscr{B}_{e}$ are semisimple, involutive, complex Banach algebras. 
Proof. Recall that the spectral radius of an element $a$ of a normed algebra $U$ is defined by $\rho(a):=\lim _{n \rightarrow \infty}\left\|a^{n}\right\|_{\ell}^{1 / n}$. In our situation, since $\|a\|_{\infty} \leq\|a\|$ for all $a$ in $\mathscr{A}_{e}$, we certainly have $\|a\|_{\infty} \leq \rho(a)$. On the other hand (3-2) implies $\sqrt{\mathscr{E}\left[a^{n}\right]} \leq n\|a\|_{\infty}^{n-1} \sqrt{\mathscr{E}[a]}$ for all $n \in \mathbb{N}^{*}$. We then have, for all $n \in \mathbb{N}^{*}$ :

$$
\left\|a^{n}\right\|=\left\|a^{n}\right\|_{\infty}+\sqrt{\mathscr{E}\left[a^{n}\right]} \leq\|a\|_{\infty}^{n}+n\|a\|_{\infty}^{n-1} \sqrt{\mathscr{E}[a]} .
$$

This implies

$$
\begin{aligned}
\rho(a) & \leq \lim _{n \rightarrow \infty}\left(\|a\|_{\infty}^{n}+n\|a\|_{\infty}^{n-1} \sqrt{\mathscr{E}}[a]\right)^{1 / n} \\
& =\lim _{n \rightarrow \infty}\|a\|_{\infty}^{(n-1) / n}\left(\|a\|_{\infty}+n \sqrt{\mathscr{E}}[a]\right)^{1 / n} \\
& =\|a\|_{\infty} .
\end{aligned}
$$

As a consequence, the set $N:=\left\{a \in \mathscr{A}_{e}: \rho(a)=0\right\}$ of the topologically nilpotent elements of $\mathscr{A}_{e}$ reduces to $\{0\}$. Since the radical $\Re$ (defined as the intersection of the kernels of all irreducible representations of $\mathscr{A}_{e}$ ) of any normed algebra is always contained in $N$ (see [Rickart 1960]), this proves that $\Re=\{0\}$ and that $\mathscr{A}_{e}$ is semisimple. The same argument applies to $\mathscr{B}_{e}$.

Corollary 3.4. The Dirichlet algebras $\mathscr{A}_{e}, \mathscr{B}_{e}$ have a unique Banach algebra topology; in particular, any norm $\|\cdot\|^{\prime}$ making $\mathscr{A}_{e}$ or $\mathscr{B}_{e}$ Banach algebras, is equivalent to the one described in Proposition 3.1:

$$
\frac{1}{k}\left(\|a\|_{\infty}+\sqrt{\mathscr{E}}[a]\right) \leq\|a\|^{\prime} \leq k\left(\|a\|_{\infty}+\sqrt{\mathscr{E}}[a]\right)
$$

for some constant $k$ and all a belonging to $\mathscr{A}_{e}$ or $\mathscr{M}_{e}$.

\section{Spectrum and $K$-theory of Dirichlet algebras}

In this section we investigate the relationship between regular Dirichlet spaces and the topology of the underlying space $X$.

Our first goal is to show that the Gelfand spectrum of the extended Dirichlet algebra $\mathscr{B}_{e}$, that is, the space of complex homomorphisms of $\mathscr{B}_{e}$, topologized with the weak*-topology of its dual space, is homeomorphic to $X$; moreover, not only does the spectral radius of an element $a \in \mathscr{B}_{e}$ coincide with its supremum norm, but the whole spectrum of $a$ is the same with respect to $\mathscr{B}_{e}$ or $C_{0}(X)$. In other words, the Gelfand transform coincides with the natural embedding of $\mathscr{B}_{e}$ into $C_{0}(X)$.

Theorem 4.1. Let $(\mathscr{E}, \mathscr{F})$ be a regular Dirichlet form on $L^{2}(X, m)$.

(i) The set $\operatorname{Rep}\left(\mathscr{B}_{e}\right)$ of all Hilbert space representations of the Banach algebra $\mathscr{B}_{e}$ coincides with the corresponding set $\operatorname{Rep}\left(C_{0}(X)\right)$ of the commutative $C^{*}$ algebra $C_{0}(X)$.

(ii) The Gelfand spectrum (or carrier space) of $\mathscr{P}_{e}$ is homeomorphic with X. 
(iii) The spectrum $\mathrm{Sp}_{\mathscr{B}_{e}}(a)$ of an element a of $\mathscr{B}_{e}$ coincides with the spectrum of a as an element of $C_{0}(X)$ :

$$
\operatorname{Sp}_{\mathscr{B}_{e}}(a)=\overline{\{a(x) \in \mathbb{C}: x \in X\}} .
$$

Proof. (i) Consider the map $\mathrm{R}: \operatorname{Rep}\left(C_{0}(X)\right) \rightarrow \operatorname{Rep}\left(\mathscr{B}_{e}\right)$ which associates to the representation $\pi$ of $C_{0}(X)$ its restriction $\mathrm{R}(\pi):=\left.\pi\right|_{\mathscr{B}_{e}}$ to the subalgebra $\mathscr{B}_{e}$. By the regularity of the Dirichlet form, $\mathscr{B}_{e}$ is uniformly dense in $C_{0}(X)$, so the map $\mathrm{R}$ is injective. Let us consider now a representation $\pi: \mathscr{B}_{e} \rightarrow \mathscr{B}(\mathscr{H})$ of $\mathscr{B}_{e}$ in a Hilbert space $\mathscr{H}$. Since $\mathscr{B}_{e}$ is an involutive Banach algebra we have $\|\pi(a)\| \leq\|a\|_{\mathscr{B}_{e}}$ for all $a \in B_{e}$. Since $\mathscr{B}(\mathscr{H})$ is a $C^{*}$-algebra, the spectral radius of $\pi(a)$ is $\|\pi(a)\|$ [Dixmier 1964, §1.3]. We then have, by Theorem 3.3:

$$
\|\pi(a)\|=\lim _{k \rightarrow \infty}\left\|\pi\left(a^{k}\right)\right\|^{1 / k} \leq \lim _{k \rightarrow \infty}\left\|a^{k}\right\|_{\mathscr{B}_{e}}^{1 / k}=\|a\|_{\infty}
$$

for all $a$ in $\mathscr{B}_{e}$. By the regularity of the Dirichlet form, $\pi$ can then be extended continuously to a representation of $C_{0}(X)$ whose restriction to $\mathscr{B}_{e}$ coincides with $\pi$. This proves that the map $R$ is surjective.

(ii) is a consequence of (i) and of the fact that the irreducible representations of $C_{0}(X), X$ being a locally compact Hausdorff space, correspond to the points of $X$, and of the characterization of the (locally compact Hausdorff) topology of the Gelfand spectrum as the weakest topology with respect to which all complex homeomorphisms are continuous.

(iii) follows from (ii) since a Dirichlet algebra is semisimple.

In case $X$ is compact and the Dirichlet form $(\mathscr{E}, \mathscr{F})$ is recurrent, the Dirichlet algebra is unital. Then Theorem 4.1(iii) tells us that an element $a$ of the Dirichlet algebra $\mathscr{B}_{e}$ is invertible in $\mathscr{B}_{e}$ if and only if it is invertible in the algebra of continuous functions $C(X)$. (In the case of noncompact spaces $X$ where $C_{0}(X)$ and $\mathscr{B}_{e}$ have no units, the latter property holds true for quasi-invertible elements; see [Rickart 1960, I.5].) Since, moreover, the existence of nontrivial invertible (matrices of) continuous functions reveals topological properties of the underlying space $X$, we may suspect that the Dirichlet algebra itself could encode some topological information such as that contained in the $K$-theory $K^{*}(X)$ of $X$. See [Atiyah 1967].

By the Arens-Eidlin-Novodvorskii theorem (see for instance [Taylor 1976]), Theorem 4.1 implies:

Theorem 4.2. Let $(\mathscr{E}, \mathscr{F})$ be a regular Dirichlet form on $L^{2}(X, m)$. Then the algebraic $K$-theory $K_{*}\left(\mathscr{B}_{e}\right)$ of the Dirichlet algebra $\mathscr{B}_{e}$ is isomorphic to the algebraic $K$-theory $K_{*}\left(C_{0}(X)\right)$ of the $C^{*}$-algebra $C_{0}(X)$, which in turn is isomorphic to the topological $K$-theory $K^{*}(X)$ of the locally compact Hausdorff space $X$. 
The isomorphism between the groups $K_{*}\left(\mathscr{B}_{e}\right)$ and $K^{*}(X)$ can be regarded as a regularization or smoothing result. For example, it implies a generalization of a classical theorem of $\mathrm{H}$. Whitney in differential topology to the effect that finitedimensional locally trivial vector bundles over smooth manifolds admits smooth structures.

Theorem 4.3. Let $(\mathscr{E}, \mathscr{F})$ be a regular Dirichlet form on $L^{2}(X, m)$. Then every finite-dimensional, locally trivial, vector bundle $E$ over $X$ admits a Dirichlet structure, that is, a compatible vector bundle atlas whose transition matrices have entries in the Dirichlet algebra $\mathscr{B}_{e}$.

The space of sections $\mathscr{B}_{e}(E, X)$ of this Dirichlet structure has a canonical Banach space topology and it is densely and continuously embedded in the space $C_{0}(E, X)$ of continuous sections of $E$ endowed with its canonical Banach space topology. $\mathscr{B}_{e}(E, X)$ is a Banach module over the Dirichlet algebra $\mathscr{B}_{e}$.

Proof. Assume, for simplicity, that $X$ is compact. By a theorem of Serre and Swan [Karoubi 1978, I.6.18], a finite dimensional, locally trivial vector bundle $E$ over $X$ is determined by the $C(X)$-module $C(E, X)$ of its continuous sections. The local triviality of $E$ implies that $C(E, X)$ is projective, and therefore of the form $p\left(C(X)^{n}\right)$ for some projection $p$ in some algebra $M_{n}(C(X))$ of matrices with continuous entries. The isomorphism between $K_{0}\left(\mathscr{B}_{e}\right)$ and $K_{0}(C(X))$ implies that there exists $n^{\prime}$ and an equivalent projection $p^{\prime}$ in $M_{n^{\prime}}\left(\mathscr{B}_{e}\right) \subset M_{n^{\prime}}(C(X))$ such that $C(E, X)=p^{\prime}\left(C(E, X)^{n^{\prime}}\right)$. The existence of an atlas having the desired properties follows from the Serre-Swan construction applied to the projection $p^{\prime}$.

As shown in [Karoubi 1978, I.6.20], the space $C(E, X)$ of continuous sections of the bundle $(E, X)$ over the compact space $X$ carries a canonical Banach space topology which depends only upon the class $[E]$ in $K^{0}(X)$. For example, it can be defined as the quotient topology associated to the surjection

$$
p: C(E, X)^{n} \rightarrow C(E, X),
$$

whenever $p$ is any projection in $M_{n}(C(X))$ which represents $[E]$ in the $K$-theory $K_{0}\left(C_{0}(X)\right) \cong K^{0}(X)$. Moreover, the quotient norms associated to equivalent projections representing $E$, give rise to equivalent Banach $C(X)$-module structures over $C(E, X)$. This is a consequence of the fact that $E$ is projective and that $C(X)$ is a $C^{*}$-algebra.

Now, having at our disposal the Dirichlet form $(\mathscr{E}, \mathscr{F})$ over $X$, we can easily construct a dense subspace $\mathscr{B}_{e}(E, X)$ of $C(E, X)$, which is the space of sections of the Dirichlet structure of $E$ defined by $(\mathscr{E}, \mathscr{F}): \mathscr{B}_{e}(E, X):=p\left(\mathscr{B}_{e}^{n}\right)$. Here $p$ is any equivalent projection in $M_{n}\left(\mathscr{B}_{e}\right)$ which represents $[E]$ in the $K$-theory $K_{0}\left(\mathscr{B}_{e}\right) \cong$ $K^{0}(X)$. 
Using projectivity and the fact that $\mathscr{B}_{e}$ is an involutive, semisimple Banach algebra, we can endow the space $\mathscr{B}_{e}(E, X)$ with a canonical topology induced by the family of equivalent quotient norms associated to the equivalent representing projections. Obviously $\mathscr{B}_{e}(E, X)$ is densely and continuously embedded in $C(E, X)$ and supports a canonical $\mathscr{B}_{e}$-module structure.

Example 4.4. Suppose that $X$ is a compact riemannian manifold, that $(\mathscr{E}, \mathscr{F})$ is the Dirichlet form defined by the Dirichlet integral $\mathscr{E}[a]=\int_{X}|\nabla a|^{2}$ over the Sobolev space $\mathscr{F}_{F}=H^{1,2}(X)$ associated to the riemannian metric over $X$, and that $E$ is the exterior bundle $\Lambda^{*}(X)$. Then our construction is equivalent to the construction of the Sobolev space $H^{1,2}\left(\Lambda^{*}(X)\right)$ of exterior forms on $X$. Notice, however, that there exist compact metric spaces that do not admit any differentiable manifold structure (e.g., fractal and other singular quotient metric spaces), but on which one can construct nontrivial regular Dirichlet forms. See, for example, [Kigami 2001; Sturm 1998].

\section{Complete regularity of regular Dirichlet space}

We now prove that regular Dirichlet algebras are completely regular. Recall that, by definition, a semisimple Banach algebra $U$ with Gelfand spectrum $X(U)$ is completely regular if, for any closed set $F \subset X(U)$ and any point $x$ not belonging to $F$, there exists $a \in U$ vanishing on $F$ and not at $x$.

Theorem 5.1. Let $X$ be a locally compact Hausdorff, metrizable space with countable base and let $(\mathscr{E}, \mathscr{F})$ be a regular Dirichlet form on $L^{2}(X, m)$. Then the Dirichlet algebra $\mathscr{B}_{e}$ is completely regular.

Proof. By Theorem 4.1, the Gelfand spectrum of $\mathscr{B}_{e}$ coincides with $X$. To prove the result we have to construct, for any closed $F \subseteq X$ and $x \notin F$, an element of $\mathscr{B}_{e}$ vanishing on $F$ but not at $x$. Since $X$ is locally compact and Hausdorff, it is a completely regular topological space, so there exists a function $a \in C_{0}(X-F)$ such that $a(x)=2$. The regularity of the Dirichlet space implies the existence of a function $b \in \mathscr{B}$ such that $\|a-b\|_{\infty}<1$. The function $c:=(b-1) \vee 0$ belongs to $\mathscr{B}$ since it is a normal contraction of $b \in \mathscr{B}$; in other words, $c=\phi \circ b$ for a suitable Lipschitz continuous, real-valued function $\phi$ of a real variable, vanishing at the origin, with Lipschitz constant at most 1 (see [Fukushima et al. 1994, Chapter 1, Theorem 1.5.3]). Since moreover $c \in C_{0}(X-F) \subset C_{0}(X)$ and $c(x)>0$, we have found an element of $\mathscr{B} \subseteq \mathscr{B}_{e}$ with the required properties.

From the theorem, the following result can be deduced applying the general theory of completely regular, semisimple Banach algebras (see [Rickart 1960]). 
Corollary 5.2. For any pair $F_{1}, F_{2}$ of disjoint closed subsets of $X$, there exists a element $a$ in the Dirichlet algebra $\mathscr{B}_{e}$ that vanishes on $F_{1}$ and assumes a constant value 1 on $F_{2}$.

Corollary 5.3 (Partition of the identity). The Dirichlet algebra $\mathscr{B}_{e}$ admits partitions of the identity: for every compact set $F \subseteq X$ and every open cover $\left\{U_{n} \subset X\right.$ : $n=1, \ldots, N\}$ of it, there exists a sequence $\left\{h_{n} \in \mathscr{B}_{e}: n=1, \ldots, N\right\}$ such that $h_{n}$ vanishes on the complement of $U_{n}$, for every $n=1, \ldots, N$, and

$$
\sum_{n=1}^{N} h_{n}=1 \quad \text { on } F .
$$

Corollary 5.4 (Local theorem). A function $a: X \rightarrow \mathbb{C}$ which coincides in suitable neighborhoods of every point of a compact set $F \subset X$ with an element of the Dirichlet algebra $\mathscr{B}_{e}$, actually coincides on $F$ with an element of $\mathscr{B}_{e}$.

Proof. For each $x \in F$ let $U_{x}$ be a neighborhood of $x$ on which $a$ coincides with an element $a_{x}$ of $\mathscr{B}_{e}$. Since $F$ is compact, there exists a finite set

$$
\left\{x_{n} \in X: n=1, \ldots, N\right\}
$$

such that $\left\{U_{x_{n}} \subset X: n=1, \ldots, N\right\}$ is a finite open cover of $F$. Let us choose a partition of the identity $\left\{h_{n} \in \mathscr{B}_{e}: n=1, \ldots, N\right\}$ subordinated to it. The element $b:=\sum_{n=1}^{N} h_{n} a_{x_{n}}$ belongs to $\mathscr{B}_{e}$ and coincides with $a$ on $F$.

\section{Ideals in Dirichlet algebras}

We now investigate some properties of the ideals of Dirichlet algebras.

Recall that for every ideal $I$ of $\mathscr{B}_{e}$, its support $F(I)$ denotes the closed subset of $X$ on which every element of $I$ vanishes. For every closed set $F \subset X$, let us define the kernel $k(F)$ of $F$ as the set of all elements of $\mathscr{B}_{e}$ vanishing on $F$. It is clearly a closed ideal of the Dirichlet algebra $\mathscr{B}_{e}$. On the other hand, denote by $J(F)$ the set of all elements of $\mathscr{B}_{e}$ vanishing in suitable neighborhoods of $F$. It is well known that $J(F)$ is minimal among the ideals whose support is $F$ and that its closure is minimal among closed such ideals. Clearly $\overline{J(F)} \subseteq k(F)$; see [Rickart 1960, II §7].

The following result was proved in [Shilov 1947] for the Dirichlet integral (1-1) on unit intervals of the real line.

Theorem 6.1. Let $(\mathscr{E}, \mathscr{F})$ be a regular Dirichlet form on $L^{2}(X, m)$. Then for every closed subset $F \subseteq X$ one has $\overline{J(F)}=k(F)$. In other words the Dirichlet algebra is an $N$-algebra (hence an $N^{*}$-algebra).

Proof. Let $a$ be an element of $k(F)$. For every $\varepsilon>0$ define $a_{\varepsilon}:=a-(-\varepsilon) \vee a \wedge \varepsilon$. Clearly $a_{\varepsilon}$ lies in $\mathscr{B}_{e}$ since it is a normal contraction of $a \in \mathscr{B}_{e}$ (see [Fukushima et 
al. 1994, Theorem 1.5.3]). Also $a_{\varepsilon}$ vanishes in the open neighborhood

$$
G_{\varepsilon}:=\{x \in X:|a(x)|<\varepsilon\}
$$

of $F$, so $a_{\varepsilon}$ lies in $J(F)$. Moreover, $a_{\varepsilon} \rightarrow a$ uniformly on $X$. In view of [Gelfand et al. 1960, $\S 36$ Theorem 1], it suffices to show that $\mathscr{E}\left[a_{\varepsilon}-a\right]=\mathscr{E}[(-\varepsilon) \vee a \wedge \varepsilon] \rightarrow 0$ as $\varepsilon \rightarrow 0$. This follows from [Fukushima et al. 1994, Theorem 1.4.2].

The next results completely describe the structure of the closed ideals of extended Dirichlet algebras.

Theorem 6.2. Let $(\mathscr{E}, \mathscr{F})$ be a regular Dirichlet form on $L^{2}(X, m)$. Then every closed ideal I of the Dirichlet algebra $\mathscr{B}_{e}$ is the kernel $k(F(I))$ of its support.

Proof. In view of Theorem 6.1, what remains to be checked is that $J(F) \subseteq I$ if $F$ is the support of $I$. Let $a$ be an element of $J(F)$ and let $G$ be the open neighborhood of $F$ on which $a$ vanishes. Then $G^{c}$ is a closed subset which is disjoint from $F$. By Corollary 5.2, there exists $b \in I$ which assumes the constant value 1 on $G^{c}$. Then by construction $a=a b \in I$ since $I$ is an ideal.

Recall that, given a regular Dirichlet form $(\mathscr{E}, \mathscr{F})$ on $L^{2}(X, m)$ and a fixed open set $G \subset X$, its part $\left(\mathscr{E}_{G}, \mathscr{F}_{G}\right)$ on $G$ is, by definition, the restriction of the quadratic form $\mathscr{E}$ on the domain $\mathscr{F}_{G}$ of those elements of $\mathscr{F}_{F}$ which vanish m-a.e. on the complement $X-G$. It is a regular Dirichlet form on the space $L^{2}(G, m)$ (see [Fukushima et al. 1994, Theorem 4.4.3]).

Corollary 6.3. Every closed ideal I of the Dirichlet algebra $\mathscr{B}_{e}(\mathscr{E}, \mathscr{F})$ is the Dirichlet algebra $\mathscr{P}_{e}\left(\mathscr{E}_{G}, \mathscr{F}_{G}\right)$ associated to the part $\left(\mathscr{E}_{G}, \mathscr{F}_{G}\right)$ of the Dirichlet form $(\mathscr{E}, \mathscr{F})$ on the complement $G:=F(I)^{c}$ of the support of $I$.

As next example shows, sometimes the quotient algebra $\mathscr{B}_{e} / I$ is itself isomorphic as a Banach algebra to a Dirichlet algebra.

Example 6.4. Let $D \subset \mathbb{R}^{n}$ be a bounded, Euclidean domain with Lipschitz boundary $\partial D$, endowed with the trace of the Lebesgue measure $\mathscr{H}^{n}$. Notice that, by the smoothness assumption, $\mathscr{H}^{n}(\partial D)=0$ so $L^{2}\left(D, \mathscr{H}^{n}\right)=L^{2}\left(\bar{D}, \mathscr{H}^{n}\right)$, where $\bar{D}:=D \cup \partial D$. With respect to the compact space $\bar{D}$, the Dirichlet integral

$$
\mathscr{E}[a]:=\int_{D}|\nabla a|^{2} d m
$$

defines a regular Dirichlet form on $L^{2}\left(\bar{D}, \mathscr{H}^{n}\right)$, finite on the Sobolev space

$$
H^{1}(D):=\left\{a \in L^{2}\left(D, \mathscr{H}^{n}\right): \nabla a \in L^{2}\left(D, \mathscr{H}^{n}\right)\right\} .
$$

Since the measure is finite, $H_{e}^{1}(D)$ and $H^{1}(D)$ coincide, so the Dirichlet algebra reduces to $H^{1}(D) \cap C(\bar{D})$. The kernel $k(\partial D)$ of the closed subset $\partial D \subset \bar{D}$ is a closed ideal in $H^{1}(D) \cap C(\bar{D})$ coinciding with the Dirichlet algebra $H_{0}^{1}(D) \cap C_{0}(D)$ 
associated with the part of the Dirichlet form on the open set $D$, i.e., the regular Dirichlet form on $L^{2}\left(D, \mathcal{H}^{n}\right)$ defined by $(6-1)$ on $H_{0}^{1}(D)$ (the latter being the Sobolev space with vanishing boundary condition, defined as the closure in $H^{1}(D)$ of the subspace of smooth functions with compact support). Again by the finiteness of the measure, we have $H_{0, e}^{1}(D)=H_{0}^{1}(D)$. To describe the quotient algebra

$$
\mathscr{L}:=\frac{H^{1}(D) \cap C(\bar{D})}{H_{0}^{1}(D) \cap C_{0}(D)},
$$

recall that the trace operator $\operatorname{tr}_{\partial D}: H^{1}(D) \rightarrow L^{2}\left(\partial D, \mathscr{H}^{n-1}\right)$ associates to each Sobolev function $a$ a well defined measurable function $\operatorname{tr}_{\partial D}(a)$ on $\partial D$, squareintegrable with respect to the $(n-1)$-dimensional Hausdorff measure $\mathscr{H}^{n-1}$. Moreover, the range of the trace operator coincides with the fractional Sobolev space

$H^{1 / 2}(\partial D):=\left\{a \in L^{2}\left(\partial D, \mathscr{H}^{n-1}\right): \frac{|a(x)-a(y)|}{|x-y|^{(n+1) / 2}} \in L^{2}\left(\partial D \times \partial D, \mathscr{H}^{n-1} \times \mathscr{H}^{n-1}\right)\right\}$,

its kernel is exactly $H_{0}^{1}(D)$ and the map is continuous with respect to the natural norm in $H^{1 / 2}(\partial D)$ (see [Brezis 1983]). Consider now the restriction

$$
t r_{\partial D}: H^{1}(D) \cap C(\bar{D}) \rightarrow H^{1 / 2}(\partial D) \cap C(\partial D)
$$

of the trace operator to the Dirichlet algebra. It is a Banach *-algebra morphism whose kernel coincides with our closed ideal. For any $b \in H^{1 / 2}(\partial D) \cap C(\partial D)$ consider the weak solution $a \in H^{1}(D)$ to the Dirichlet problem

$$
\left\{\begin{aligned}
-\Delta a+a & =0 \text { on } D \\
a=b & \text { on } \partial D .
\end{aligned}\right.
$$

Because the boundary data is assumed to be continuous, and by the regularity theory of the weak solutions of the Dirichlet problem (see [Brezis 1983]), we have $a \in H^{1}(D) \cap C(\bar{D})$, so the trace operator above is surjective too. This shows that the quotient algebra $\mathscr{L}$ can be identified with the Dirichlet algebra associated to the Dirichlet form on $\partial D$ :

$$
\mathscr{E}_{\partial D}[a]:=\int_{\partial D \times \partial D} \frac{|a(x)-a(y)|^{2}}{|x-y|^{(n+1)}} \mathscr{H}^{n-1} \times \mathscr{H}^{n-1}(d x, d y)
$$

on the fractional Sobolev space $H^{1 / 2}(\partial D)$ and $+\infty$ otherwise in $L^{2}\left(\partial D, \mathscr{H}^{n-1}\right)$.

In case $D$ is the unit disk in the plane $\mathbb{R}^{2}$, the Dirichlet space at the boundary is just the Douglas integral (see [Fukushima et al. 1994]). 


\section{Applications to representations and comparisons of strongly local Dirichlet forms}

In this section we apply the previous analysis to show that a strongly local, regular Dirichlet form is determined by its Dirichlet algebra. Consider, on the space $\mathbb{D}(X, m)$ of all Dirichlet forms on the space $L^{2}(X, m)$, the semi-order $\preceq$ defined by

$$
\text { (7-1) } \quad \mathscr{E}_{1} \preceq \mathscr{E}_{2} \Longleftrightarrow \mathscr{E}_{2}[a] \leq c \mathscr{E}_{1}[a] \text { for all } a \in L^{2}(X, m) \text { and some } c>0 \text {. }
$$

By Lejan's domination principle [1978], if the forms are strongly local the order relation is equivalent, in terms of energy measures, to the condition $\mu_{\langle a\rangle}^{2} \leq c \mu_{\langle a\rangle}^{1}$ for all $a \in \mathscr{F}_{1}$; that is, $\mu_{\langle a\rangle}^{2}$ is absolutely continuous with respect to $\mu_{\langle a\rangle}^{1}$ and the Radon-Nikodym derivative is $\mu_{\langle a\rangle}^{1}$-a.e. uniformly bounded above by a constant $c$.

Two Dirichlet forms are said to be quasi-equivalent, in symbols $\mathscr{E}_{1} \simeq \mathscr{E}_{2}$, if $\mathscr{E}_{1} \preceq \mathscr{E}_{2}$ and $\mathscr{E}_{2} \preceq \mathscr{E}_{1}$, that is, if

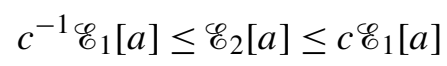

for some constant $c>0$ and all $a \in L^{2}(X, m)$.

Notice that, implicitly, if $\mathscr{E}_{1} \preceq \mathscr{E}_{2}$ then $\mathscr{F}_{1} \subseteq \mathscr{F}_{2}$. We are going to see that the opposite implication is also true for strongly local, regular Dirichlet forms.

Theorem 7.1. Let $\mathscr{E}_{1}, \mathscr{E}_{2} \in \mathbb{D}(X, m)$ be strongly local, regular Dirichlet forms on $(X, m)$. Then

$$
\mathscr{E}_{1} \preceq \mathscr{E}_{2} \Longleftrightarrow \mathscr{F}_{1} \subseteq \mathscr{F}_{2} \Longleftrightarrow \mathscr{B}_{e}^{1} \subseteq \mathscr{R}_{e}^{2} .
$$

In particular they are quasi-equivalent, $\mathscr{E}_{1} \simeq \mathscr{E}_{2}$, if and only if they have the same domain $\mathscr{F}_{1}=\mathscr{F}_{2}$ or the same Dirichlet algebra $\mathscr{B}_{e}^{1}=\mathscr{B}_{e}^{2}$.

Proof. Suppose that $\mathscr{B}_{e}^{1} \subseteq \mathscr{B}_{e}^{2}$. Our assumptions allow us to consider the identity map from $\mathscr{B}_{e}^{1}$ to $\mathscr{R}_{e}^{2}$ as a morphism of algebras. Since, by Theorem $3.3, \mathscr{B}_{e}^{2}$ is a semisimple Banach algebra, the identity map is continuous by [Rickart 1960, Theorem 2.5.17]. We then have

$$
\|a\|_{\infty}+\sqrt{\mathscr{\mathscr { C }}_{2}[a]} \leq c\left(\|a\|_{\infty}+\sqrt{\mathscr{E}_{1}[a]}\right) \quad \text { for } a \in \mathscr{B}_{e}^{1},
$$

for some constant $c>0$. In turn we have

$$
\|a\|_{\infty}^{2}+\mathscr{E}_{2}[a] \leq c\left(\|a\|_{\infty}^{2}+\mathscr{E}_{1}[a]\right) \quad \text { for } a \in \mathscr{B}_{e}^{1},
$$

for some other constant $c>0$. Consider now the two families of normal contractions defined by $\phi_{\lambda}(s):=\lambda^{-1} \sin (\lambda s), \psi_{\lambda}(s):=\lambda^{-1}(1-\cos (\lambda s))$ for all $s \in \mathbb{R}$ and $\lambda \neq 0$. Noticing that $\left|\phi_{\lambda}^{\prime}(s)\right|^{2}+\left|\psi_{\lambda}^{\prime}(s)\right|^{2}=1$ for all $s \in \mathbb{R}$ and $\lambda \neq 0$ and applying 
the chain rule for the energy measures $\mu_{\langle\cdot\rangle}^{i}, i=1,2$ representing $\mathscr{E}_{1}$ and $\mathscr{E}_{2}$ (see [Fukushima et al. 1994]), we have the identities

$$
\begin{aligned}
\mathscr{E}_{i}\left[\phi_{\lambda}(s)\right]+\mathscr{E}_{i}\left[\psi_{\lambda}(s)\right] & =\int_{X} d \mu_{\left\langle\phi_{\lambda}(a)\right\rangle}^{i}+\int_{X} d \mu_{\left\langle\psi_{\lambda}(a)\right\rangle}^{i} \\
& =\int_{X}\left|\phi_{\lambda}^{\prime}(a)\right|^{2} d \mu_{\langle a\rangle}^{i}+\int_{X}\left|\psi_{\lambda}^{\prime}(a)\right|^{2} d \mu_{\langle a\rangle}^{i} \\
& =\int_{X} d \mu_{\langle a\rangle}^{i}=\mathscr{E}_{i}[a]
\end{aligned}
$$

for all $\mathscr{B}_{e}^{1}, \lambda \neq 0$ and $i=1,2$. Combining (7-2) and (7-3) and noticing that

$$
\lim _{\lambda \rightarrow+\infty}\left\|\phi_{\lambda}(a)\right\|_{\infty}=\lim _{\lambda \rightarrow+\infty}\left\|\psi_{\lambda}(a)\right\|_{\infty}=0
$$

we have, for a suitable constant $c>0$, that $\mathscr{E}_{2}[a] \leq c \mathscr{E}_{1}[a]$ for all $a \in \mathscr{B}_{e}^{1}$. We conclude by noticing that $\mathscr{B}_{e}^{1} \cap \mathscr{F}_{1}$ is a form core for $\mathscr{E}_{1}$ and that $\mathscr{E}_{2}$ is lower semicontinuous, so the same bound holds true for all $a \in \mathscr{F}_{1}$.

Applying Lejan's domination principle we have:

Theorem 7.2. Let $\mathscr{E}_{1}, \mathscr{E}_{2} \in \mathbb{D}(X, m)$ be strongly local, regular Dirichlet forms on $(X, m)$ and denote by $\mu_{\langle\cdot\rangle}^{1}, \mu_{\langle\cdot\rangle}^{2}$ their energy measures. Then

$$
\begin{array}{ll}
\mathscr{F}_{1} \subseteq \mathscr{F}_{2} \Longleftrightarrow \mu_{\langle a\rangle}^{2} \leq c \mu_{\langle a\rangle}^{1} & \text { for some } c>0 \text { and all } a \in \mathscr{F}_{1} ; \\
\mathscr{F}_{1}=\mathscr{F}_{2} \Longleftrightarrow c^{-1} \mu_{\langle a\rangle}^{1} \leq \mu_{\langle a\rangle}^{2} \leq c \mu_{\langle a\rangle}^{1} & \text { for some } c>0 \text { and all } a \in \mathscr{F}_{1}=\mathscr{F}_{2} .
\end{array}
$$

Let $D$ be a Euclidean domain, with its Lebesgue measure $m$. A result of Beurling and Deny [1959] describes all strongly local, regular Dirichlet forms on $L^{2}(D, m)$ that have the space of test functions $C_{c}^{\infty}(D)$ as a core. Their energy measures have, for $a \in C_{c}^{\infty}(D)$, the form

$$
\mu_{\langle a\rangle}=\sum_{i, j=1}^{n} \partial_{i} a \partial_{j} a \mu^{i j}
$$

for a system of Radon measures $\mu^{i j}$ satisfying $\mu^{i j}=\mu^{j i}$ for all $i, j=1, \ldots, n$ and $\sum_{i, j=1}^{n} \xi_{i} \xi_{j} \mu^{i j} \geq 0$ for all $\left(\xi_{1}, \ldots, \xi_{n}\right) \in \mathbb{R}^{n}$.

We will see that if more information is known about the form domain, a more detailed description of the Dirichlet from and its energy measures is possible. The following result answers the questions considered in the introduction.

Corollary 7.3. Let $D \subseteq \mathbb{R}^{n}$ be a bounded domain and consider the Sobolev space $H_{0}^{1}(D)$. Then any strongly local, regular Dirichlet form $(\mathscr{E}, \mathscr{F})$ on $L^{2}(D, m)$ whose 
domain $\mathscr{F}$ contains $H_{0}^{1}(D)$ has the representation

$$
\mathscr{E}[a]=\sum_{i, j=1}^{n} \int_{D} g^{i j} \partial_{i} a \partial_{j} a d m \quad a \in H_{0}^{1}(D)
$$

for a symmetric matrix $\left[g^{i j}\right]_{i, j}$ of measurable functions on D satisfying

$$
0 \leq \sum_{i, j=1}^{n} \xi_{i} \xi_{j} g^{i j} \leq c\|\xi\|^{2}
$$

$m$-a.e. on $D$, for some $c>0$ and all $\left(\xi_{1}, \ldots, \xi_{n}\right) \in \mathbb{R}^{n}$.

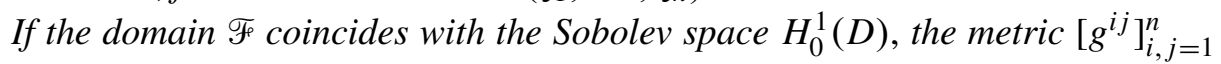
appearing in the representation (7-4) is uniformly quasi-conformal to the Euclidean one:

$$
c^{-1}\|\xi\|^{2} \leq \sum_{i, j=1}^{n} \xi_{i} \xi_{j} g^{i j} \leq c\|\xi\|^{2}
$$

$m$-a.e. on $D$, for some $c>0$ and all $\left(\xi_{1}, \ldots, \xi_{n}\right) \in \mathbb{R}^{n}$.

Proof. By assumption, the domain $\mathscr{F}$ of $\mathscr{E}$ contains the Sobolev space $H_{0}^{1}(D)$, which is the domain of the strongly local, regular Dirichlet form on $D$ given by the Dirichlet integral

$$
\mathscr{E}_{D}[a]=\int_{D}|\nabla a|^{2} d m
$$

Then $H_{0}^{1}(D) \subseteq \mathscr{F}$ implies, by Theorem 7.1, that $\mathscr{E}_{D} \preceq \mathscr{E}$. By Theorem 7.2 we then have the bound

$$
\sum_{i, j=1}^{n} \partial_{i} a \partial_{j} a \mu^{i j} \leq c|\nabla a|^{2} d m,
$$

for all $a \in H_{0}^{1}(D)$ and then also for all $a$ in the space $\left(C(D) \cap H^{1}(D)\right)_{\text {loc }}$ of functions locally contained in the Dirichlet algebra $C(D) \cap H^{1}(D)$. We can then apply (7-7) to the coordinate functions $a_{i}(x):=x_{i}, i=1, \ldots, n$, obtaining that $\mu^{i i}:=\mu_{\left\langle a_{i}\right\rangle} \leq c m$ for some $c>0$ and all $i=1, \ldots, n$. The Schwartz rule for energy measures (see [Fukushima et al. 1994]) then implies that $\mu^{i j} \leq \mathrm{cm}$ for some other $c>0$, so a measurable matrix with the property (7-5) is guaranteed to exist. If the domain $\mathscr{F}$ coincides with $H_{0}^{1}(D)$, Theorem 7.2 then yields

$$
c^{-1}|\nabla a|^{2} d m \leq \sum_{i, j=1}^{n} \partial_{i} a \partial_{j} a \mu^{i j} \leq c|\nabla a|^{2} d m,
$$


for some $c>0$ and for all $a \in H_{0}^{1}(D)$. From this the uniformly elliptic bound (7-6) follows easily.

Whenever the boundary $\partial D$ is sufficiently smooth, we may, by slightly modifying the above argument, also consider reflecting boundary conditions in place of the absorbing ones.

Corollary 7.4. Let $D$ be a bounded Lipschitz domain with closure $\bar{D}$ and consider the Sobolev space $H^{1}(D)$. Then, any strongly local, Dirichlet form on $L^{2}(D, m)$, whose domain $\mathscr{F}$ contains $H^{1}(D)$, has the representation (7-4) for all a $\in H^{1}(D)$, for a suitable symmetric matrix $\left[g^{i j}\right]_{i, j=1}^{n}$ of measurable functions on $D$ satisfying the bound (7-5).

If the domain of $\mathscr{E}$ coincides with the Sobolev space $H^{1}(D)$, the metric $\left[g^{i j}\right]_{i, j=1}^{n}$ in the representation (7-4) satisfies the bound (7-6).

Proof. By assumption, the domain $\mathscr{F}$ of $\mathscr{E}$ contains the Sobolev space $H^{1}(D)$, which is the domain of the strongly local, regular Dirichlet form on $\bar{D}$ given by the Dirichlet integral

$$
\mathscr{E}_{D}[a]=\int_{D}|\nabla a|^{2} d m .
$$

See [Fukushima et al. 1994, Example 4.4.1]. Then $H^{1}(D) \subseteq \mathscr{F}$ implies, by Theorem 7.1, that $\mathscr{E}_{D} \preceq \mathscr{E}$. By Theorem 7.2 we then have the bound

$$
\sum_{i, j=1}^{n} \partial_{i} a \partial_{j} a \mu^{i j} \leq c|\nabla a|^{2} d m,
$$

for all $a \in H^{1}(D)$ and then for all $a$ in the space $\left(C(\bar{D}) \cap H^{1}(D)\right)_{\text {loc }}$ of functions locally contained in the Dirichlet algebra $C(\bar{D}) \cap H^{1}(D)$. Applying the argument above we conclude the proof.

\section{Concluding remarks}

We conclude by pointing out some considerations and problems which, in our opinion, arise naturally from the preceding analysis.

Dirichlet forms, commutative or not, arise in various fields of mathematics: in connection with Markov processes and Markov semigroups in classical and quantum probability; in connection with semibounded, self-adjoint operators representing the Hamiltonian of a physical system in mechanics, quantum mechanics and quantum field theory; and as quadratic forms of the Laplace-Beltrami operator or Dirac's laplacian in riemannian geometry. In each one of these frameworks, the contraction property $\mathscr{E}[a \wedge 1] \leq \mathscr{E}[a]$ translates a precise symmetry property of the energy $\mathscr{E}$ of a probabilistic, physical or geometric nature. 
The results of Section 7 then show that a knowledge of the set $\mathscr{F}$ of configurations of finite energy imposes severe restrictions on the structure of the allowable energy forms $\mathscr{E}$ : specifically, in the strongly local case, $\mathscr{F}$ determines the uniformly quasi-conformal class of $\mathscr{E}$. This is a consequence of the fact that, normed in a natural way, spaces of bounded functions of finite energy realize semisimple Banach algebras.

On the other hand, the description of the ideal structure of the Dirichlet algebra $\mathscr{B}$ led us to prove that the $K$-theory of the compact Hausdorff space $X$ can be detected from the $K$-theory of $\mathscr{B}$. A geometric way to look at this result is that Dirichlet forms on a compact, Hausdorff $X$, give rise to Dirichlet structures on finite-dimensional, locally trivial vector bundles over $X$.

In [Cipriani and Sauvageot 2003] we showed that regular Dirichlet forms, commutative or not, give rise to a canonical first-order differential calculus, namely, a closable derivation $\partial$ in symmetric tangent Hilbert bimodule $\mathscr{H}$, by which the energy form can be represented as $\mathscr{E}[a]=\|\partial a\|_{\mathscr{H}}^{2}$. Notice that $\partial$ is naturally defined precisely on the Dirichlet algebra $\mathscr{B}$.

In this respect it should be interesting to extend the differential calculus from the Dirichlet algebra $\mathscr{B}$ to the bimodule $\mathscr{B}(E, X)$ of bounded, finite energy sections of finite, locally trivial vector bundles $E$ over $X$.

Finally, two problems of functional analytic nature arise naturally: i) which uniformly dense subalgebras of $C_{0}(X)$ arise as (extended) Dirichlet algebras of regular Dirichlet forms on $X$ ? ii) which subalgebras of $L^{\infty}(X, m)$ arise as the (extended) weak Dirichlet algebras of regular Dirichlet forms on $X$ ?

\section{Acknowledgements}

I thank Sergio Albeverio and Gianfausto Dell' Antonio for interesting discussions about the subject of this work. I thank also A. M. Chebotarev for providing me with a copy of [Shilov 1947].

\section{References}

[Albeverio 2003] S. Albeverio, "Theory of Dirichlet forms and applications", pp. 1-106 in Lectures on probability theory and statistics (Saint-Flour, 2000), Lecture Notes in Math. 1816, Springer, Berlin, 2003. MR 2005a:60120 Zbl 1043.60063

[Atiyah 1967] M. F. Atiyah, K-theory, Benjamin, New York, 1967. Reprinted Addison-Wesley, Redwood City (CA), 1989. MR 36 \#7130 Zbl 0159.53302

[Beurling and Deny 1959] A. Beurling and J. Deny, "Dirichlet spaces", Proc. Nat. Acad. Sci. U.S.A. 45 (1959), 208-215. MR 21 \#5098 Zbl 0089.08201

[Brezis 1983] H. Brezis, Analyse fonctionnelle: Théorie et applications, Masson, Paris, 1983. MR 85a:46001 Zbl 0511.46001 
[Cipriani 1997] F. Cipriani, "Dirichlet forms and Markovian semigroups on standard forms of von Neumann algebras”, J. Funct. Anal. 147:2 (1997), 259-300. MR 98i:46067 Zbl 0883.47031

[Cipriani and Sauvageot 2003] F. Cipriani and J.-L. Sauvageot, "Derivations as square roots of Dirichlet forms", J. Funct. Anal. 201:1 (2003), 78-120. MR 2004e:46080 Zbl 1032.46084

[Dixmier 1964] J. Dixmier, Les $C^{*}$-algèbres et leurs représentations, Cahiers Scientifiques 29, Gauthier-Villars, Paris, 1964. 2nd edition, 1969. MR 30 \#1404 Zbl 0152.32902

[Fukushima et al. 1994] M. Fukushima, Y. Ōshima, and M. Takeda, Dirichlet forms and symmetric Markov processes, de Gruyter Studies in Mathematics 19, de Gruyter, Berlin, 1994. MR 96f:60126 Zbl 0838.31001

[Gelfand et al. 1960] I. M. Gel'fand, D. A. Răkov, and G. E. Shilov, Коммутативные нормированные колца, Gosudarstv. Izdat. Fiz.-Mat. Lit., Moscow, 1960. Translated as Commutative normed rings, Chelsea, New York, 1964. MR 23 \#A1242 Zbl 0134.32102

[Karoubi 1978] M. Karoubi, K-theory, Grundlehren der Math. Wissenschaften 226, Springer, Berlin, 1978. MR 58 \#7605 Zbl 0382.55002

[Kigami 2001] J. Kigami, Analysis on fractals, Cambridge Tracts in Mathematics 143, Cambridge University Press, Cambridge, 2001. MR 2002c:28015 Zbl 0998.28004

[Lejan 1978] Y. Lejan, "Mesures associées à une forme de Dirichlet: applications", Bull. Soc. Math. France 106:1 (1978), 61-112. MR 81c:31014 Zbl 0393.31008

[Ma and Röckner 1992] Z. M. Ma and M. Röckner, Introduction to the theory of (nonsymmetric) Dirichlet forms, Universitext, Springer, Berlin, 1992. MR 94d:60119 Zbl 0826.31001

[Rickart 1960] C. E. Rickart, General theory of Banach algebras, Van Nostrand, Princeton, NJ, 1960. MR 22 \#5903 Zbl 0095.09702

[Shilov 1947] G. Shilov, О регулярных нормированных колцах [On regular normed rings], Trud. Mat. Inst. Steklov. 21, Izd. Ak. Nauk SSSR, Moscow, 1947. MR 9,596a Zbl 0045.38201

[Silverstein 1976] M. L. Silverstein, Boundary theory for symmetric Markov processes, Lecture Notes in Math. 516, Springer, Berlin, 1976. MR 56 \#9708 Zbl 0331.60046

[Sturm 1998] K. T. Sturm, "Diffusion processes and heat kernels on metric spaces", Ann. Probab. 26:1 (1998), 1-55. MR 99b:31008 Zbl 0936.60074

[Taylor 1976] J. L. Taylor, "Topological invariants of the maximal ideal space of a Banach algebra", Advances in Math. 19:2 (1976), 149-206. MR 53 \#14134 Zbl 0323.46058

[Wermer 1960] J. Wermer, "Dirichlet algebras", Duke Math. J. 27 (1960), 373-381. MR 22 \#12405 Zbl 0099.32002

Received October 1, 2003. Revised August 10, 2005.

\section{FABIO CIPRIANI}

DiPARTIMENTO Di MATEMATICA

POLITECNICO Di MiLANO

PIAZZA LEONARDO DA VINCI 32

20133 Milano

ITALY

fabio.cipriani@mate.polimi.it 